\title{
INHALATION OF METHICILLIN FOR THE CONTROL OF STAPHYLOCOCCAL CROSS-INFECTION
}

\author{
SUSAN GoldFARB
}

Clare Hall Hospital, South Mimms, Barnet.

THE danger of staphylococcal cross-infection is an ever present problem in a thoracic unit, and especially in a thoracic surgical unit. The reason is that many patients admitted to such a unit are heavy carriers of staphylococci in their sputa.

One of our surgical wards was closed twice because of a high cross-infection rate. After the first closure we introduced the following measures, which are usually recommended for preventing cross-infection:-

1. Broom sweeping was discontinued and suction machines were introduced.

2. The woollen blankets were replaced by openweave broadloom cotton blankets and were changed on the beds of infected patients once a week.

3. Bed linen was changed daily, and was carefully collected into bins. No counting of linen took place on the ward.

4. All wound dressing was done in the treatment room, and the soiled dressings were placed into paper bags, and then into bins with self-closing lids.

5. The number of cubicles was increased, and all infected cases were nursed in these cubicles.

6. Naseptin was introduced as a nasal prophylactic, and was administered to the patient on admission, and then twice daily under the supervision of the ward sister.

7. Antibiotics were only used when the sensitivity of the infecting organism was available.

8. Weekly nasal swabs were taken from all staff, and even those with minor skin sepsis were excluded from duty.

However, though the cross-infection rate was low for a time after the ward was re-opened, it soon rose again in spite of these well known measures. A second ward closure became inevitable, and it was realised that a new approach was needed.

The method of methicillin spraying was chosen, mainly because this approach was found most effective by Elek and Fleming (1960a) in totally eradicating Staph. aureus from a maternity unit.

The aim of this method is to establish a fine aerosol of methicillin particles, which are inhaled by the carriers of staphylococci. The particles dissolve in the nasal and respiratory secretion, and attack the staphylococci at their very source.

A total of $10 \mathrm{~g}$. of methicillin dissolved in $200 \mathrm{ml}$. of water was sprayed daily in four divided amounts spaced throughout the day. This works out at $0.3 \mathrm{~g}$. of methicillin per 1,000 c. ft. per day.

The result of applying this method was a great success. After a few weeks of methicillin spraying total eradication of staphylococcal cross-infection was achieved. (Fig. 1).

This result was not due to case selection, as infected patients were admitted to the ward as before. Once the full effectiveness of the drug became established it was found that staphylococci disappeared from the noses and sputa of newly admitted patients within a week. The only source of staphylococci remaining on the ward were those patients with a deep seated lesion, present in the patient before admission, such as an abscess or an empyema.

It was routine to test all staphylococci for methicillin sensitivity and no resistant strains were found. In fact, it became completely impossible to isolate any staphylococci.

It was not expected that any resistant strains would be found, firstly because they did not occur in the trial of Elek and Fleming (1960a, $1960 \mathrm{~b})$, and secondly on theoretical grounds. Resistant staphylococci would have to be either ones producing an enzyme capable of destroying methicillin, or those with intrinsic drug tolerance. However there is no indication so far, that staphylococci causing significant destruction of methicillin exist, and those naturally occurring methicillin resistant staphylococci are very rare, and possess a relatively slight degree of resistance. It is prob- 


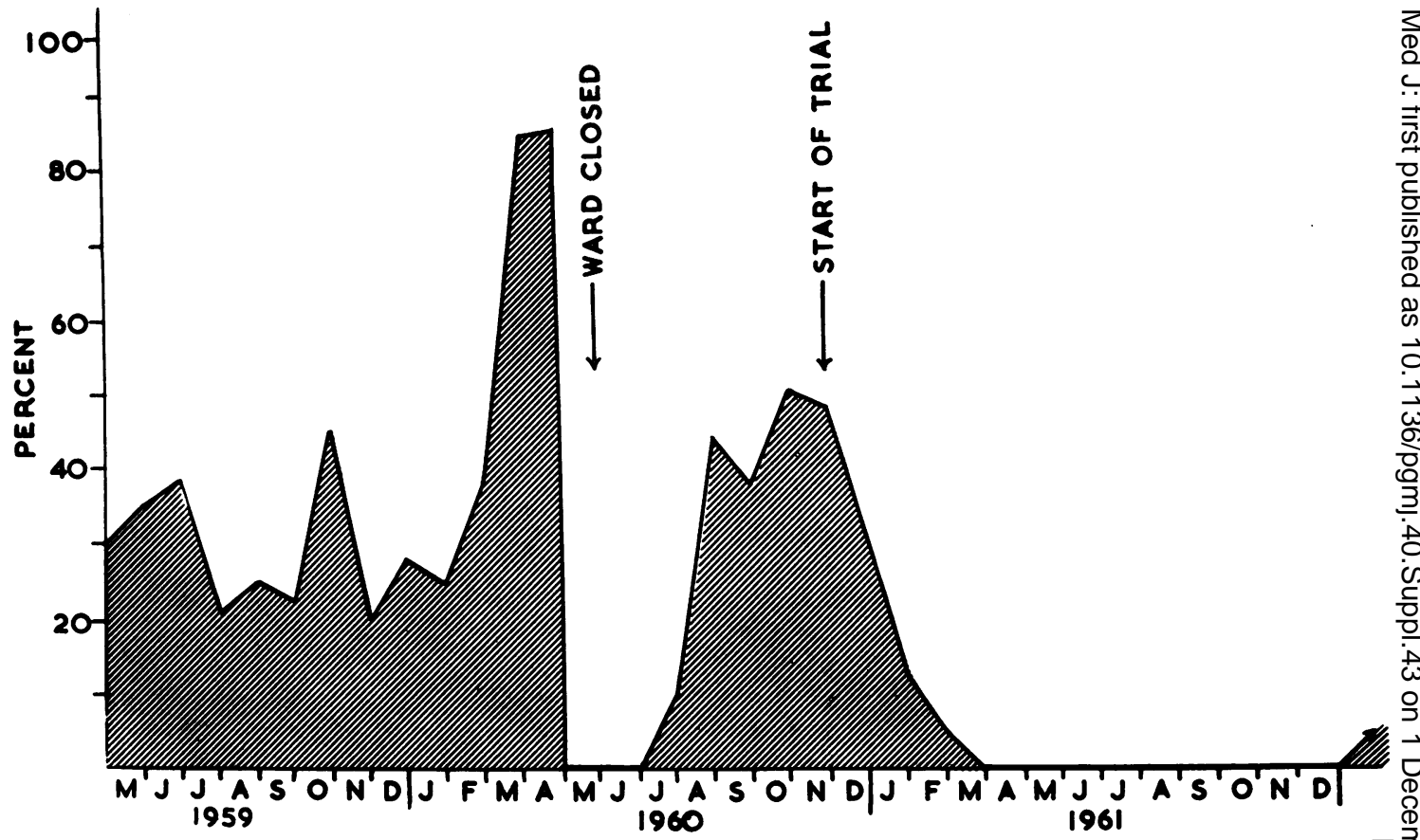

Fig. 1.-Shows the cross-infection rate month by month, expressed as a percentage. There was a maintained high level of cross-infection, which rose to a peak in April 1960, and we had to close the ward. As soon as the ward was re-opened cross-infection reappeared, and reached a level of $50 \%$ in December 1960 . At that time the trial of methicillin spraying was started, and there was a dramatic reduction in cross-infection. No more cases occurred during that year to the end of the period of the trial. 224 patients were admitted during the period to the ward.

able that the concentration of methicillin at the site of inhalation is too high to allow these strains to multiply.

No hypersensitivity reactions were observed, though it was known, that a few patients were hypersensitive to penicillin.

Encouraged by these results in the prophylactic field, we decided to use drug spraying as a therapeutic measure. This was tried on a ward containing many chronic bronchitics. These patients are often infected with staphylococci or Gram-negative organisms. Systematic administration of antibiotics is at times ineffective in such patients.

We tried spraying with neomycin first, in the hope of eliminating both Gram-positive and Gram-negative infections. The procedure was the same as for methicillin spraying.

Very few of the infections cleared, and after the second week of spraying resistant organisms started to emerge. In two patients hypersensitivity reactions occurred, with severe rash and blood eosinophilia, whereupon spraying was discontinued.

We then selected patients infected with resistant staphylococci and exposed them spraying with methicillin, but under slight different conditions.

The air of a small room, $12 \mathrm{ft}$. $\times 5 \mathrm{ft} . \times 7 \mathrm{f} \overrightarrow{\vec{b}}$, was sprayed with methicillin three times a da甲, and the patients spent an hour in this room after each spraying.

From the standpoint of clearing infections, this was successful. Within a week the sputa were converted from mucopurulent to th: mucoid.

However, the small enclosed room produced a sense of suffocation in many of the dyspnoeic patients. For this reason we had to stop using it.

We then changed over to individual sprayin of patients suffering from staphylococc bronchitis. This was done by means of a faces mask. Three ml. of a $10 \%$ solution was cono verted into an aerosol with the aid of a Wrights nebuliser attached to an oxygen cylinder. The patient inhaled the methicillin through the mask three times a day, for ten minutes each time.

Fifteen patients have been treated by the 
method up to now. In all of them freedom from staphylococci was achieved, and they improved clinically.

\section{Conclusion}

The first part of these studies shows, that the inhalation of methicillin particles leads to the total eradication of staphylococci as a source of cross-infection. This result cannot be achieved by any of the methods previously described. In spite of predictions to the contrary, the emergence of drug resistance and hypersensitivity phenomena have not been found.
In the second part of these studies, that is the treatment of staphylococcal bronchitis by the inhalation of methicillin, a successful result was achieved.

Finally we have made some financial comparisons, and these have shown that the various methods of spraying are considerably cheaper, than is the cost of maintaining infected patients in hospital.

\section{REFERENCES}

Elek, S. D., and Fleming, P. C. (1960a), Lancet, i, 1009.

Elek, S. D., and Fleming, P. C. (1960b), Ibid., ii, 569 .

\title{
CLINICAL EXPERIENCES WITH THE PROPHYLACTIC AND THERAPEUTIC USES OF THE NEW PENICILLINS IN CARDIOVASCULAR-RENAL DISEASES
}

\author{
ELLARD M. Yow
}

Infectious Disease Section, Department of Internal Medicine, Baylor University College of Medicine, and the Infectious Disease Laboratory, Methodist Hospital, Houston, Texas.

THE treatment of a staphylococcal infection complicating the implantation of cardiovascular prostheses has almost always failed unless the prostheses were removed. Even if the prostheses were removed and a new one implanted in an infected field, the procedure was usually unsuccessful. The greatest success has been in those cases in which it was possible to remove the prosthesis, adequately treat the infection, and re-implant the graft in a sterile field. However, removal of a graft in the aorta or a prosthetic heart valve is not always compatible with life for sufficient periods of time to allow adequate treatment of the infection.

While infection of cardiovascular prostheses is not common (though it was reported to be as high as $10 \%$ at one clinic) (Geraci, Dale and McGoon, 1963), when it does occur it is usually life threatening. The difficulties encountered in the management of these infections when they do occur made us consider more seriously chemoprophylaxis as a possible approach to this problem. Consequently, three years ago a programme was instituted in which all patients having cardiovascular surgery in two of the four hospitals in the Texas Medical Center doing this type of surgery were given methicillin 6 grams daily and streptomycin
1 gram daily for two days before and four days following surgery. Those patients giving a history of allergy to penicillin were excluded from the study.

A rather complex statistical analysis of the results of this study is now being carried out, but since several thousand cardiovascular operations are involved it will require some time for completion. It is of interest, however, that the information already available reveals that there have been no proved cases of serious staphylococcal infections in the methicillin treated group, whereas there have been several deaths among the control groups in whom infections played a major role.

The use of methicillin prophylactically has not prevented all infections. There have been several patients who developed Gram-negative bacillary infections, notably pseudomonas, but none of these have been as difficult to handle as have the staphylococcal infections.

The same prophylactic régime was used in four patients receiving kidney homotransplantations and two additional patients known to be allergic to penicillin received cephalothin rather than methicillin. There have been no infections in this group of patients.

While it seems likely at this stage that the 